\title{
Le phosphore
}

\author{
Phosphorus \\ par P. Balland \\ Mission d'Inspection Spécialisée de l'Environnement
}

Phosphorus is a key element of the plants development in aquatic environments. This processus, called eutrophication when the excess of plants becomes a problem, is concerning an increasing number of aquatic mediums in our world. After an inventory of phosphorus characteristics, the CORPEN has lauched an action program in order to get under control phosphorus disposal from agriculture.

\section{I $\square$ LE PHOSPHORE : QUELQUES RAPPELS}

Corps simple légèrement ambré, très inflammable (" le phosphore, ce feu étrange entre tous puisqu'il se conserve sous I'eau ", G. Bachelard), lumineux dans l'obscurité (" phosphore = qui porte la lumière "), le phosphore est, sous un grand nombre d'espèces chimiques, une brique essentielle du développement de tous les organismes vivants [1].

Chez l'homme par exemple, où il est présent au taux de 1,1 à $1,2 \mathrm{~g} / \mathrm{kg}$ de son poids (dont $85 \%$ dans le squelette et les dents), il joue un rôle premier dans trois fonctions essentielles [2] :

- réservoir et transfert d'énergie nécessitée par la " veille" métabolique et l'activité physique, mettant en jeu notamment I'A.T.P. (adénosine-5 triphosphate),

- synthèse des acides aminés et des protéines et transmission de l'information (code génétique), comme élément constitutif des molécules d'ARN et d'ADN respectivement,

- édification des tissus durs, squelette et dents, dont le développement et la maintenance sont à l'origine des besoins, par l'alimentation, et des pertes, par les excreta, les plus importants.

Les besoins moyens sont, chez l'enfant, de $0,5 \mathrm{~g} /$ jour, chez l'adulte, de 0,8 à $1 \mathrm{~g} /$ jour selon la corpulence. Ils s'élèvent à $1,2 \mathrm{~g} /$ jour chez la femme enceinte et allaitante.

Les végétaux, algues et végétaux supérieurs (macrophytes), expriment à l'encontre du phosphore des exigences physiologiques tout aussi impératives que chez l'homme et les animaux.

Ils ont besoin de cet élément pour leur développement et leur croissance, en particulier pour l'accomplissement de la fonction qui leur est spécifique de l'assimilation chlorophyl- lienne, par laquelle ils bénéficient de la propriété de synthétiser leur propre matière, à partir de l'énergie lumineuse et d'une série d'ingrédients essentiels : c'est la photosynthèse dont l'équation fondamentale est donnée ci-après [3].

$106 \mathrm{CO}_{2}+16 \mathrm{NO}_{3}+\mathrm{HPO}_{4}+122 \mathrm{H}_{2} \mathrm{O}+18 \mathrm{H}+$ + oligoéléments + énergie lumineuse

$$
\frac{\mathrm{C}_{106} \mathrm{H}_{263} \mathrm{O}_{110} \mathrm{~N}_{16} \mathrm{P}_{1}}{\text { matériau végétal }}+\frac{\Downarrow}{138 \mathrm{O}_{2}}
$$

La prise en compte de $\mathrm{NH}_{4}$, au lieu de $\mathrm{NO}_{3}$, comme source d'azote ne change pas les proportions des différents constituants du matériau végétal.

En poids, le phosphore représente $0,9 \%$ de ce dernier. Cela signifie que $1 \mathrm{~g}$ de phosphore assimilé équivaut à $115 \mathrm{~g}$ de matériau végétal synthétisé $(1 \mathrm{~g}$ d'azote $=16 \mathrm{~g}$ de matière végétale).

En termes de rapport N/P, important à considérer dans la stratégie de maîtrise, on constate qu'il est égal à 16 en atomes, alors qu'il vaut 7 en poids.

Cependant, il s'agit d'une équation moyenne et en pratique, chaque espèce algale et végétale exprime des exigences particulières quant à la valeur optimale de ce rapport, qui peut varier de 7 à $45[4]$. On notera en particulier que les ulves, macroalgues vertes principales responsables des proliférations végétales des côtes bretonnes et des lagunes languedociennes, présentent un rapport N/P optimal très bas, de l'ordre de 7. 


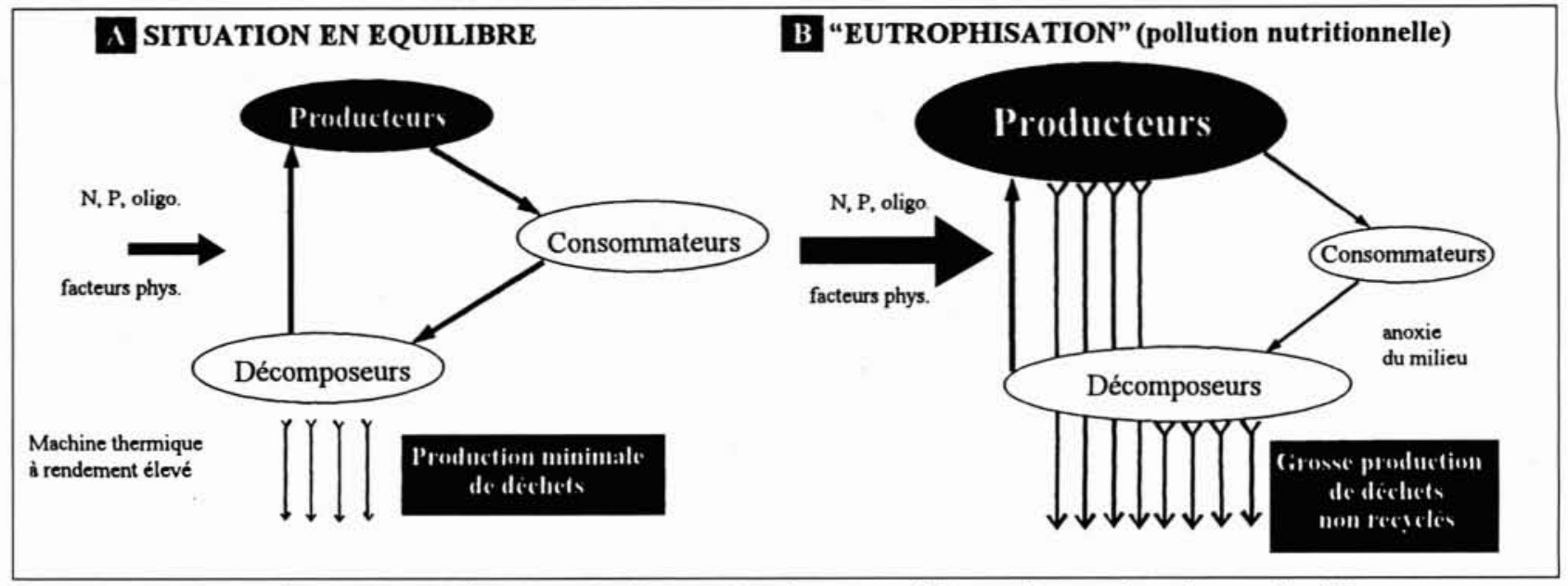

1. Représentation schématique de l'eutrophisation, par référence à une situation en équilibre.

Pour l'essentiel, l'assimilation par les algues et les macrophytes ne mobilise qu'une seule espèce chimique de phosphore, l'ion orthophosphate soluble, alors qu'il est présent dans la nature sous une grande variété de formes. Cette espèce chimique particulière sera assimilée en premier en proportion de sa présence, les autres espèces doivent être rendues biodisponibles (et bioassimilables) par des processus biochimiques d'hydrolyse plus ou moins efficaces selon les conditions du milieu et leur propre nature.

\section{I IMPACTS DU PHOSPHORE SUR LES MILIEUX AQUATIQUES ET LES USAGES DE L'EAU}

\subsection{La manifestation de l'eutrophisation}

Les algues et macrophytes sont naturellement présents dans tous les milieux aquatiques. Premier maillon de l'édifice biologique, ils en sont un compartiment essentiel, celui des producteurs, en association avec les compartiments complémentaires des consommateurs et des décomposeurs.

Ils sont à la base de la chaîne alimentaire, consomment du gaz carbonique, produisent de l'oxygène et jouent un rôle d'abri pour les invertébrés et les poissons (macrophytes et algues fixées).

La disponibilité à l'excès de l'azote et du phosphore (parmi d'autres causes potentielles) brise les mécanismes d'autorégulation de l'édifice biologique par la stimulation du compartiment primaire qui n'est plus contrôlé. Son développement prend alors des proportions considérables qui vont au-delà des capacités d'assimilation par les consommateurs et les décomposeurs. C'est le phénomène de l'eutrophisation (fig.1), appellation impropre quoique universellement utilisée, car elle est dotée d'une connotation plutôt positive (“ qui nourrit bien ”). Il serait plus approprié de parler de dystrophie ou de pollution nutritionnelle, dès lors que l'équilibre initial de l'édifice biologique est rompu. Une caractéristique importante en est la production en masse de

Tableau l : Formes végétales rencontrées dans les eaux soumises à l'eutrophisation.

\begin{tabular}{|c|c|c|}
\hline forme végétale & aspect & milieux concernés \\
\hline $\begin{array}{c}\text { phytoplancton (algues } \\
\text { microscopiques en } \\
\text { suspension) }\end{array}$ & $\begin{array}{c}\text { coloration de l'eau (verte, brune, rouge } \\
\text { selon les espèces) } \\
\text { réduction de la transparence }\end{array}$ & $\begin{array}{c}\text { plans d'eau (lacs, lagunes), } \\
\text { cours d'eau lents et parfois } \\
\text { milieu marin }\end{array}$ \\
\hline $\begin{array}{c}\text { diatomées fixées } \\
\text { (algues microscopiques) }\end{array}$ & $\begin{array}{c}\text { dépôt sur le fonds des cours } \\
\text { d'eau, galets glissants }\end{array}$ & $\begin{array}{c}\text { cours d'eau lents } \\
\text { ou rapides }\end{array}$ \\
\hline $\begin{array}{c}\text { algues filamenteuses } \\
\text { fixées }\end{array}$ & $\begin{array}{c}\text { grandes algues, envahissant } \\
\text { parfois toute la masse d'eau } \\
\text { Cladophores : longs filaments } \\
\text { de plusieurs mètres }\end{array}$ & cours d'eau \\
\hline $\begin{array}{c}\text { macroalgues vertes } \\
\text { (ulves) }\end{array}$ & aspect de laitues & $\begin{array}{c}\text { lagunes languedociennes } \\
\text { estrans bretons }\end{array}$ \\
\hline $\begin{array}{c}\text { végétaux supérieurs } \\
\text { (potamots, renoncules, } \\
\text { myriophylles, etc.) }\end{array}$ & allure de végétaux terrestres & $\begin{array}{c}\text { tronçons de cours d'eau } \\
\text { lents à fonds déposés et } \\
\text { plans d'eau peu profonds }\end{array}$ \\
\hline
\end{tabular}




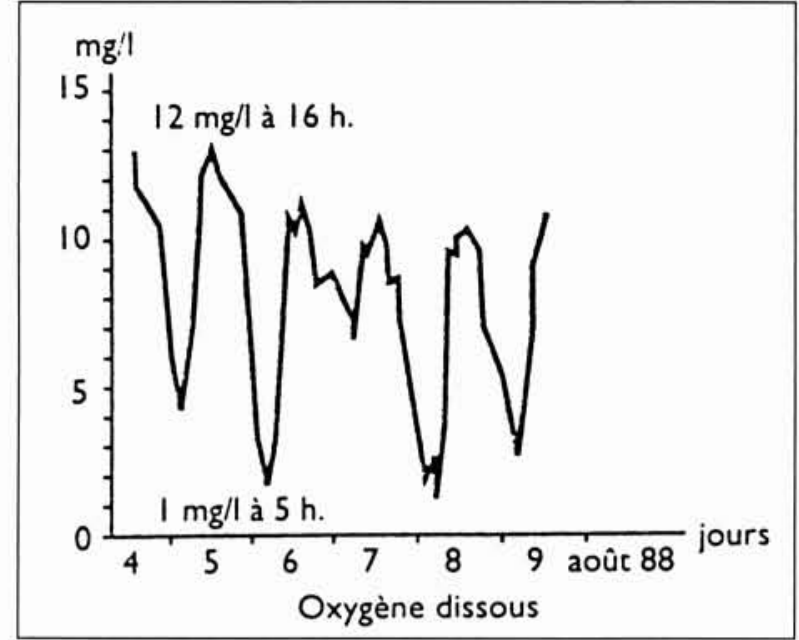

2. Variation de l'oxygène dissous et du pH sur le Doubs à l'aval d'Arçon en 1988. (Proliférations des algues en Franche-Comté. Observatoire de l'eau de la région Franche-Comté déc. 1994).

déchets organiques non recyclés [5].

L'eutrophisation revêt des formes multiples, selon les milieux, dont le tableau 1 donne un résumé simplifié.

Les problèmes aigus d'eutrophisation ne sont dus qu'à un nombre limité d'espèces.

\subsection{Les principaux désordres dus à l'eutrophisation}

Ils affectent les milieux aquatiques eux-mêmes, et leur fonctionnement initial, et une série d'usages de l'eau et des produits de l'eau. On en donne ci-après une liste non hiérarchisée, en termes d'incidence.

- Exacerbation du cycle nycthéméral de l'oxygène dissous :

C'est la manifestation la plus caractéristique. Elle est due à une très forte production diurne (par photosynthèse) et une très forte consommation nocturne (par respiration) d'oxygène par la biomasse végétale, pouvant aller jusqu'à l'anoxie avec des conséquences importantes sur les biocénoses, piscicoles notamment.

- Déstabilisation du statut d'acido-basicité (pH) du milieu : par déplacement de l'équilibre calco-carbonique (milieux calcaires en particulier) engendré par la photosynthèse et la consomation du $\mathrm{CO}_{2}$ de l'eau, il en résulte une forte augmentation du $\mathrm{pH}$ lors des épisodes de production diurne.

La figure 2 donne une illustration de ces incidences sur le Doubs à l'aval d'Arçon.

Si les conditions de température s'y prêtent (notamment si la température est élevée), l'augmentation du $\mathrm{pH}$ peut favoriser la transformation de l'azote ammoniacal ionisé en ammoniac gazeux $\left(\mathrm{NH}_{3}\right)$ ou ammoniaque $\left(\mathrm{NH}_{4} \mathrm{OH}\right)$ extrêmement toxiques à très faibles doses à l'encontre des peuplements piscicoles.

- Production de composés toxiques : par les Dinoflagellés, notamment en milieu saumâtre et marin. Les coquillages qui concentrent ces composés peuvent devenir impropres à la consommation, faisant alors l'objet d'une interdiction provisoire de commercialisation ou de collecte : exemple du symptôme du D.S.P. : Diarrheic Shellfish Poison, dont souffre périodiquement la conchyliculture.

- Destruction d'habitat : par colmatage des fonds des cours d'eau, et destruction du milieu d'évolution naturelle de certains invertébrés ou de zones de frai des poissons.

- Pollution organique différée : A leur mort, les végétaux et les algues subissent une minéralisation à l'origine d'un appauvrissement en oxygène dissous de l'eau pouvant aller jusqu'à l'anoxie. Au milieu d'origine se substitue alors un milieu radicalement différent où s'expriment librement les phénomènes d'anaérobiose totalement dominés par le compartiment bactérien et pouvant, notamment en milieu stagnant, aller jusqu'à la production de gaz tels que $\mathrm{NH}_{4}, \mathrm{H}_{2} \mathrm{~S}$ et $\mathrm{CH}_{4}$, produits par la décomposition anaérobie de la matière organique (exemple de la malaïgue des étangs languedociens due à la décomposition des ulves). La dépréciation écosystémique qui en résulte est majeure ainsi que l'incidence sur l'activité conchylicole en lagune saumâtre.

- Dégradation de la qualité de l'eau distribuée : due aux difficultés rencontrées lors des traitements de potabilisation des eaux touchées par une eutrophisation phytoplanctonique : - colmatage des filtres et des grilles de prise d'eau,

- variations importantes et rapides de la qualité de l'eau $(\mathrm{pH}$, $\mathrm{NH}_{4}$, matière organique ...) rendant difficile l'adaptation en continu des traitements nécessaires,

- risques de production de substances indésirables comme par exemple les géosmines qui communiquent à l'eau un goût de moisi difficile et coûteux à traiter.

- Atteinte directe à la santé humaine et animale :

En eau douce, en été, se développent parfois des souches de Cyanobactéries qui produisent des hépatotoxines ou des neurotoxines. Les risques induits vis-à-vis de la santé humaine et animale, quoique mal connus, se distinguent néanmoins des désordres précédemment évoqués par la gravité potentielle de leurs conséquences. Il est important d'en avoir une évaluation précise.

- Altération de la valeur esthétique et du potentiel ludique du milieu :

Influencé par l'eutrophisation, il n'attire plus, n'engage plus à la pratique des activités de loisir aquatique, ce qui a des répercussions sur l'économie locale, touchée en terme de fréquentation touristique.

Difficiles à chiffrer, les dommages à l'activité économique sont importants : de l'ordre de deux milliards de F/an. S'y ajoute la dépréciation patrimoniale non quantifiable.

\subsection{Un exemple d'actualité : les macroalgues vertes du littoral breton [6].}

Sur l'ensemble du littoral breton, les sites où les ulves sont présentes en accumulations significatives étaient au nombre de 45 en 1997. Ils ont été classés en cinq catégories selon l'importance pondérale estimée de la biomasse (tableau 2).

Tableau 2 : Classification des sites affectés par les macroalgues vertes.

\begin{tabular}{|c|c|}
\hline Biomasse fraîche estimée & Indice \\
\hline de 4000 à $8000 \mathrm{t}$ & 8 \\
de 2000 à $4000 \mathrm{t}$ & 4 \\
de 1000 à $2000 \mathrm{t}$ & 2 \\
de 500 à $1000 \mathrm{t}$ & 1 \\
moins de $500 \mathrm{t}$ & 0,5 \\
\hline
\end{tabular}




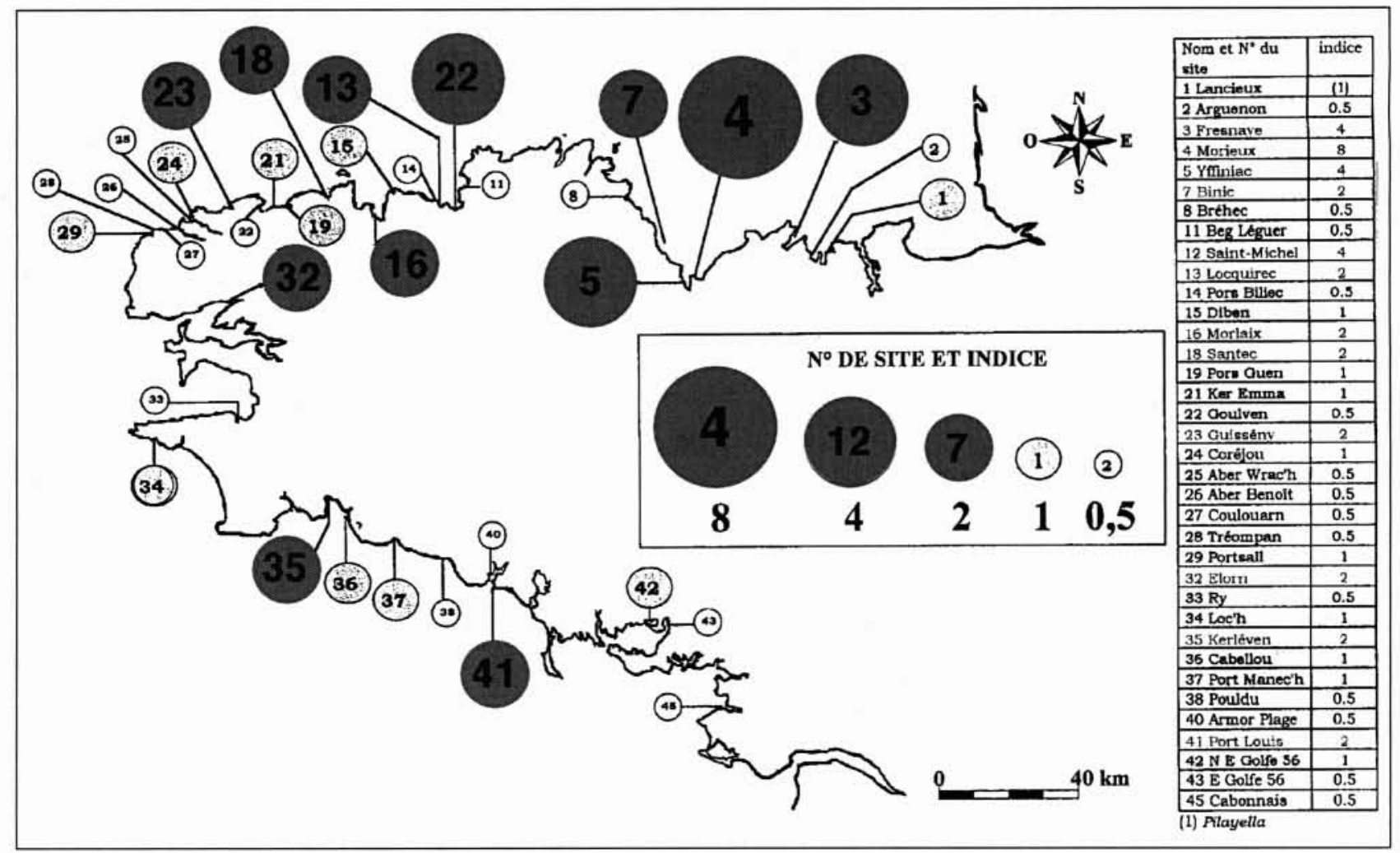

3. Recensement des proliférations algales juillet-août 1997.

Les Côtes d'Armor et le Finistère sont les départements les plus concernés. L'Ille-et-Vilaine n'est pas touchée par le phénomène de marée verte et le Morbihan assez peu (fig. 3 ). A ces sites, il convient d'en ajouter dix autres, où se sont développées des espèces autres que les ulves : Enteromorpha sp. le plus souvent (algue verte) et Pilayella à Lancieux (algue brune).

Ils sont répartis dans les trois départements des Côtes d'Armor, du Finistère et du Morbihan, les biomasses y atteignant des valeurs peu élevées

L'année 1998 a confirmé ces résultats en les amplifiant parfois, comme à Binic (site 7 de la figure 3 ) où une prolifération considérable d'ulves, dépassant l'indice 8 , s'est pro- duite. La conséquence principale en est la dépréciation des sites affectés par le tourisme. En 1998 également, l'interdiction provisoire de consommation des coquillages a été prononcée par les Préfets de Loire-Atlantique et des Côtes d'Armor.

\section{III $\square$ ORIGINES DU PHOSPHORE}

\subsection{Considération préliminaire}

Avant de passer en revue, par grandes masses, les diverses origines du phosphore que l'on rencontre dans le milieu naturel, il y a lieu d'abord de mettre en exergue deux notions fortes qui permettent de mieux comprendre les

Tableau 3 : Inventaire des principales origines diffuses et ponctuelles du phosphore.

\begin{tabular}{|l|l|l|}
\hline \multirow{2}{*}{ Type d'activité et d'origine } & \multicolumn{2}{|c|}{ Phosphore provenant de } \\
\cline { 2 - 3 } & \multicolumn{1}{|c|}{ sources ponctuelles } & \multicolumn{1}{c|}{ sources diffuses } \\
\hline 1. Ville et village (domestique) & $\begin{array}{l}\text { - tout-à-légout et puits perdus } \\
\text { - station d'épuration (STEP) } \\
\text { - domestique dispersé }\end{array}$ & $\begin{array}{l}\text { - ruissellement urbain } \\
\text { - dépôt d'ordures } \\
\text { - marécages drainés ou remblayés }\end{array}$ \\
\hline 2. Industrie & - rejet direct & - ruissellement urbain \\
\hline 3. Agriculture & $\begin{array}{l}\text { - stockage concentré (fumier, lisier, ensilage) } \\
\text { - nettoyage des locaux } \\
\text { - rejets directs, élevages }\end{array}$ & $\begin{array}{l}\text { - stockage dispersé } \\
\text { - sols cultivés et prairies } \\
\text { - routes et chemins }\end{array}$ \\
\hline 4. Zone “ naturelle " & $\begin{array}{l}\text { il est transmis indépendamment des périodes } \\
\text { de ruissellement, au rythme des activités } \\
\text { humaines. Il est généralement émis par un } \\
\text { tuyau, à l'exception des sources agricoles }\end{array}$ & $\begin{array}{l}\text { il passe par, dans, ou sur le sol et il est } \\
\text { transféré lors de périodes pluvieuses }\end{array}$ \\
\hline
\end{tabular}


bases d'une stratégie de maîtrise d'une part, et de mieux relativiser les responsabilités respectives des diverses origines dans la manifestation de l'eutrophisation d'autre part.

1 - le phosphore est naturellement présent dans le lithosphère, mais à des taux très faibles $(0,11 \%)$, hors les gisements constitués de roches phosphatées : collophanite, vivianite et surtout apatite sous diverses associations, exploitées en tant que telles. Sous ces formes " naturelles ", le phosphore n'est quasiment pas mobilisable par les végétaux.

2 - le phosphore n'a pas, à l'inverse de l'azote, d'espèce chimique volatile susceptible de jouer le rôle de source, à partir de l'atmosphère, ou de puits, par volatilisation atmosphérique.

Il en résulte deux conséquences pratiques fondamentales :

I - la stratégie de maitrise de l'eutrophisation (voir chapitre IV ci-après) s'est historiquement concentrée sur le phosphore et rien ne justifie de la remettre en cause.

2 - quel que soit le site, plus ou moins durable, de sa localisation à un instant donné (tissus animaux et végétaux, milieux aquatique et terrestre...) le phosphore considéré à l'échelle du territoire national représente un stock en augmentation permanente et inexorable.

En effet, l'accroissement démographique, l'augmentation du cheptel et des rendements agricoles, les exigences sanitaires, le développement industriel... sont autant de "faits " socio-économiques stimulant les importations de phosphore (et concourant à l'augmentation des stocks en place) et que ne compensent pas les " sorties" hors du territoire national, représentées essentiellement par les exportations de produits agricoles, animaux et végétaux.

La stratégie de la maîtrise du phosphore s'inscrit donc dans ce contexte très particulier, elle ne saurait en aucun cas s'apparenter à son élimination (et donc à une baisse du stock). On mesure dans ces conditions à quel point sa conduite est délicate (voir chapitre IV ci-après).

\subsection{Origines du phosphore}

Ce qui précède permet une certaine hiérarchie des origines du phosphore, que l'on examine ci-après en termes de tonnages annuels respectifs, sachant que toutes, peu ou prou, contribuent à accroître un stock en place déjà considérable. En effet, il est possible de distinguer entre les origines :

- en circuit ouvert, c'est-à-dire qui sollicitent sans cesse une importation à partir, notamment, de fabrications industrielles. Il s'agit pour l'essentiel :

- des produits lessiviels : lavage du linge, des textiles, de la vaisselle, entretien des locaux...

- de l'alimentation humaine, et surtout animale, pour sa partie importée,

- des fabrications industrielles consommatrices de phosphore,

- de la fertilisation des cultures par engrais industriel.

Même si l'on vise à la réduction des flux (cas des lessives) ou à l'optimisation du couple fourniture/besoins (cas de l'alimentation animale ou de la fertilisation des cultures), ces diverses origines ont le point commun de consommer du phosphore importé et de restituer au milieu quasiment tout (lessives) ou partie (cultures, animaux) de l'importation.

- en circuit pseudo fermé (il n'existe pas de circuit fermé strict) avec recyclage partiel. Il s'agit essentiellement du phospore produit et consommé à la ferme pour lequel on cherche à optimiser le couple " production de déjections animales/valorisation par les fourrages ". C'est également à cette activité qu'on demande de recycler (valoriser) les sousproduits provenant de l'épuration des effluents domestiques (boues), ce qui ne peut qu'accroître la complexité de la conduite de l'exploitation agricole.

Ces caractéristiques doivent amener à considérer particulièrement les origines du phosphore, allant au-delà de leur quantification en flux annuels, d'autant que les modes de transfert aux milieux sensibles sont extrêmement diversifiés, ainsi qu'en atteste le tableau 3 [7].

D'une manière générale, on rencontre sur un bassin donné l'ensemble des origines de ce tableau en proportions différenciées. L'activité agricole y est représentée mais sa contribution ne doit pas être résumée aux seuls transferts diffus. L'équation " agricole $=$ diffus " est non seulement simplificatrice mais erronée. Il semble même que la maîtrise du " ponctuel agricole" (élevages) soit plus pressante et prioritaire en cas d'excédent de production de déjections par rapport aux possibilités de valorisation.

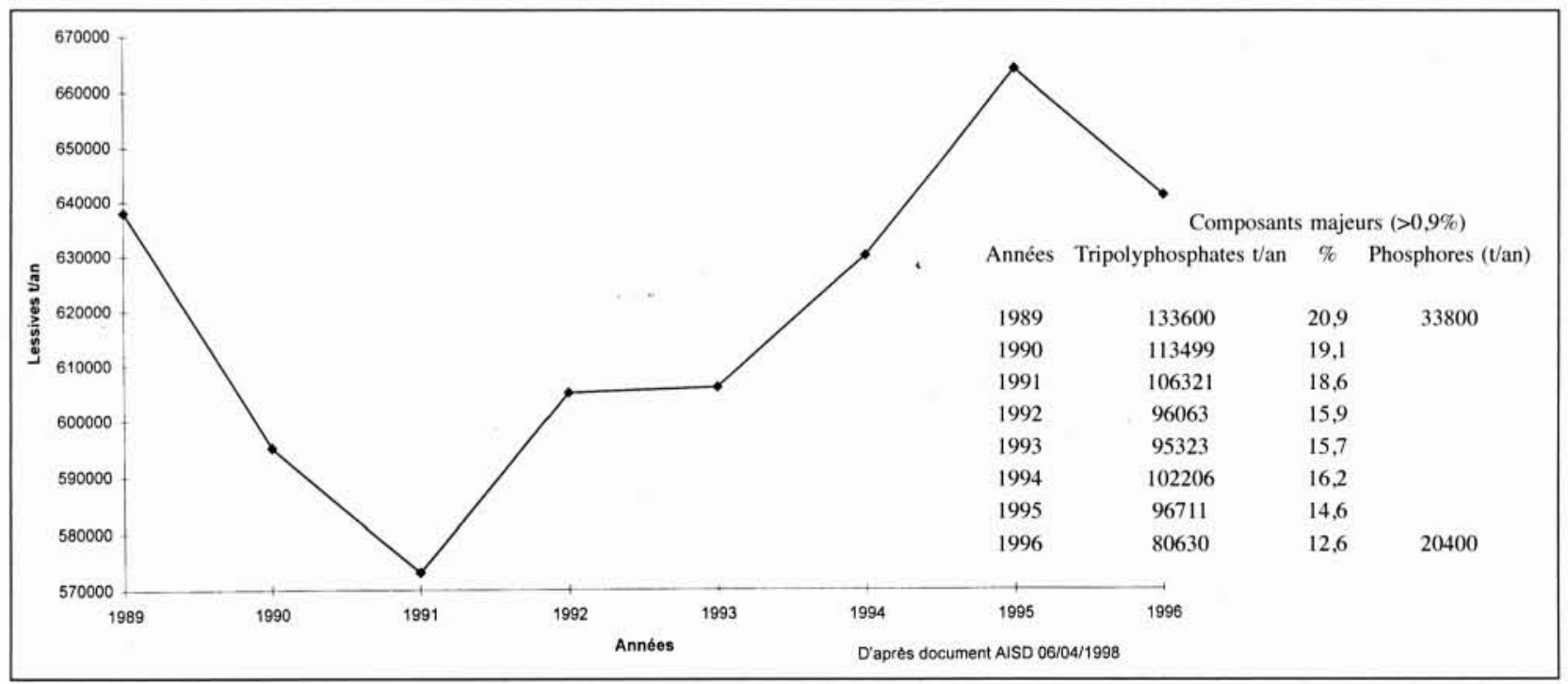

4. Evolution de la quantité de lessives utilisées en France de 1989 à 1996. 
On donne ci-après quelques chiffres d'émission annuelle, par source, qui doivent être vus à la lumière et ce qui est dit précédemment.

\subsubsection{Origine humaine (métabolisme)}

Bon an mal an, l'homme excrête de 1,2 à 1,6 g/jour de phosphore (en moyenne $1,5 \mathrm{~g} / \mathrm{j}$ soit $0,55 \mathrm{~kg} / \mathrm{an}$ ).

Rapporté à la population nationale, ce chiffre équivaut à une émission totale de... $33000 \mathrm{t} / \mathrm{an}$ à la fois incompressible et à forte biodisponibilité.

\subsubsection{Origine lessivielle (source : document AISD du 4 juin 1998)}

En 1996, le phosphore des produits lessiviels consommés en France s'est élevé à 20400 t (fig. 4).

En baisse notable (environ $40 \%$ en 8 ans conformément aux accords passés) le phosphore des lessives n'en est pas moins très biodisponible. Comme dans le cas du phosphore métabolique, une part appréciable rejoint le milieu naturel.

S'y ajoute le phosphore des composants mineurs, et notamment les phosphonates, en légère augmentation sur la période : 1140 t/an en $1989 ; 1512$ t/an en 1996.

\subsubsection{Origine industrielle}

Elle provient essentiellement de 3 secteurs d'activité :

Au total 20 à 30000 t/an [5].

- I'industrie mécanique, dont le traitement de surface,

- l'agroalimentaire,

- quelques industries chimiques.

\subsubsection{Origine engrais industriels [7] : source UNIFA 1997 (tableau 4).}

\section{Période 1979-1980}

Période 1995-1996

$866000 \mathrm{t}$ P/an $450000 \mathrm{t}$ P/an

Comme pour les lessives, on note (fig.5) une sensible baisse de la fertilisation à l'ha (diminution de près de $50 \%$ en un peu plus de 20 ans). Elle se situe aux environs de $40 \mathrm{~kg} / \mathrm{ha} \mathrm{SAU}$ de P2O5, soit $17,5 \mathrm{~kg} / \mathrm{ha} \mathrm{SAU} \mathrm{de} \mathrm{P,} \mathrm{avec} \mathrm{une}$ inégale répartition selon les régions (fig.6).

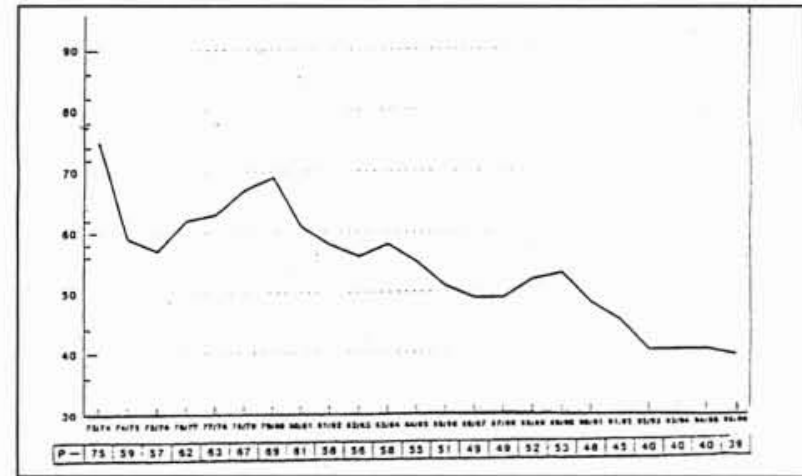

5. Evolution de la fertilisation à l'ha SAU $(\mathrm{kg} / \mathrm{ha}$ de P2O5) entre 1973 et 1996.

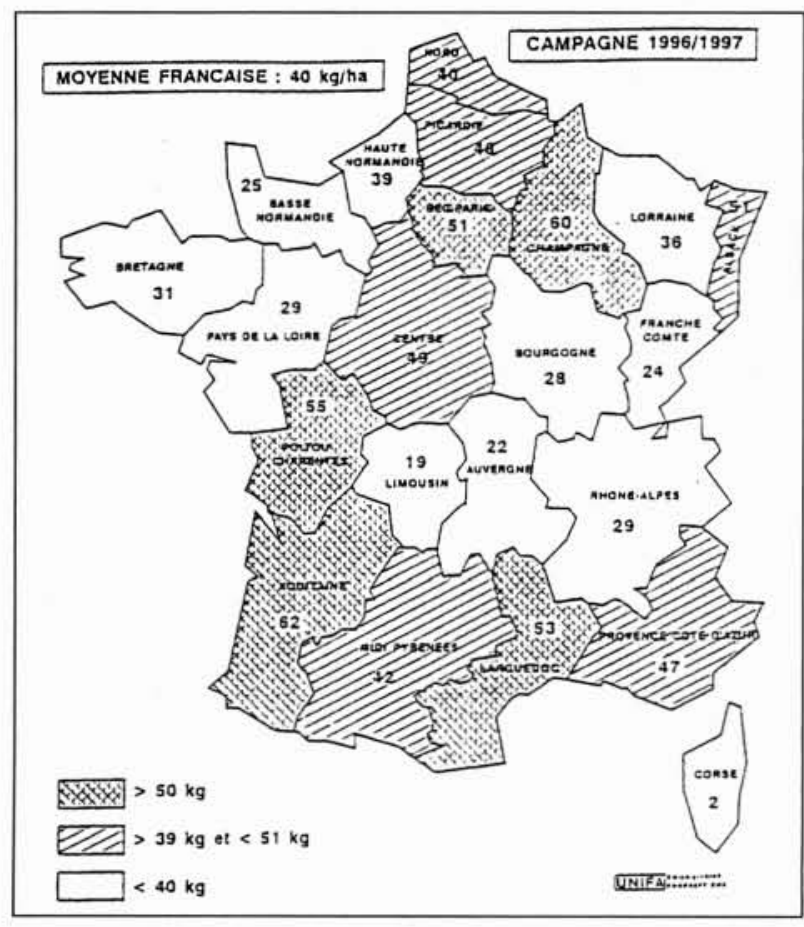

6. Consommation par région ( $\mathrm{kg} / \mathrm{ha} \mathrm{P2O5})$.

Tableau 4: Tonnages de P205 par catégories d'engrais phosphates.

\begin{tabular}{|l|c|c|c|c|}
\hline & $1979-1980$ & $1995-1996$ & $\begin{array}{c}\text { Variations } \\
\text { tonnes }\end{array}$ & $\begin{array}{c}\text { Variations } \\
\%\end{array}$ \\
\hline Scories & 224100 & 11900 & -212200 & $-94,7$ \\
\hline Superphosphates & 197200 & 134000 & -65200 & $-33,1$ \\
\hline Phosphates naturels & 73200 & 9700 & -63500 & $-86,7$ \\
\hline $\begin{array}{l}\text { Divers dont amende- } \\
\text { ments phosphatés }\end{array}$ & 1300 & 13000 & +11700 & $+900,-$ \\
\hline Scories potassiques & 67500 & 29300 & -38200 & $-56,6$ \\
\hline Superpotassiques & 254300 & 210000 & -44300 & $-17,4$ \\
\hline Phosphopotassiques & 111700 & 34900 & -76800 & $-68,8$ \\
\hline Divers PK & 203500 & 28800 & -174700 & $-85,8$ \\
\hline $\begin{array}{l}\text { Binaires NP dont } \\
\text { phosphate d'amoniaque }\end{array}$ & 161900 & 215400 & +53500 & $+33,0$ \\
\hline Ternaires & 689200 & 344300 & -344900 & $-50,0$ \\
\hline Total & 1983900 & 1031300 & -952600 & $-48,0$ \\
\hline
\end{tabular}


3.2.5 Origine animale (déjections): Source Gueguen INRA 1996 [7] - environ 300000 t/an (fig. 7).

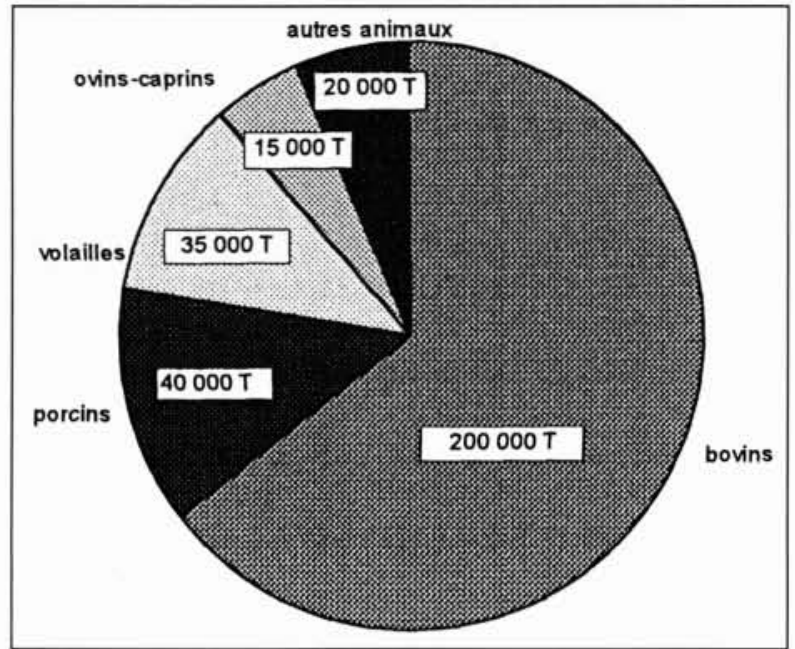

7. Répartition du phosphore des déjections animales produites annuellement en France.

Cet apport varie peu, en attendant l'effet sur sa composition de la mise en œuvre des références en matière d'alimentation animale.

\subsubsection{Commmentaires}

On note le poids équivalent des 3 premières origines, au total 73000 à $83000 \mathrm{t} / \mathrm{an}$, fortement nocives dans la mesure où pour leur plus grande part, elles sont assimilables à des pollutions ponctuelles, rejoignant directement le milieu aquatique et constituées d'espèces chimiques facilement biodisponibles.

$80000 \mathrm{t}$ de $\mathrm{P}$ suffisent à la synthèse potentielle de $9,2 \mathrm{Mt}$ de matériau végétal.

Les origines agricoles (engrais et déjections), d'un ordre de grandeur dix fois supérieur aux 3 premières, n'ont cependant pas une incidence en proportion de leur importance compte tenu de ce qui a été dit sur les modes de transfert et le recyclage. Elles ne peuvent toutefois pas être exemptées d'une responsabilité potentielle, probablement assez compa- rable à celle des 3 premières origines. La préoccupation première en ce qui les concerne, est celle des rejets directs d'élevages, incriminés notamment dans le cas des marées vertes bretonnes.

Cela milite pour une stratégie de maîtrise, en ce qu'elle vise la réduction des apports au milieu, s'adressant à toutes les origines, au nom d'un principe à valeur d'application globale de solidarité de tous les usagers et acteurs économiques dans la lutte contre l'eutrophisation.

On notera pour finir que cet ensemble de flux en provenance de ces diverses origines se surajoute annuellement au stock considérable d'ores et déjà en place, dans les sols et les fonds des rivières, des lacs et du littoral (notion d'entropie du stock de phosphore en place évoquée au chapitre 3.1.)

\section{IV $\square$ CONCLUSION : LES FONDEMENTS D'UNE STRATÉGIE DE MAITRISE}

\subsection{Principes}

Puisque l'élimination du phosphore (par exemple par la voie atmosphérique, elle ne serait d'ailleurs qu'un transfert) n'est pas possible et dans le contexte décrit d'accroissement du stock en place, la stratégie de maîtrise ne peut que se circonscrire aux axes opérationnels ci-après (non exclusifs l'un de l'autre).

1. abaisser le niveau des importations : c'est le point fort des stratégies respectives de fabrication de produits lessiviels, de l'alimentation animale (pour sa partie importée), de la fertilisation des cultures par les engrais de synthèse.

2. soustraire les milieux sensibles aux effets des rejets directs en phosphore, par intervention volontaire sur leur composition.

3. améliorer les performances de l'exploitation agricole, en termes de recyclage des intrants phosphorés (notion de circuit pseudo fermé ci-avant évoquée).

4. confiner les flux de phosphore avant qu'ils parviennent aux milieux aquatiques.

5. traiter au cas par cas les sédiments des rivières et des lacs

6. aménager et entretenir les cours d'eau, dans le sens de l'amoindrissement des effets des facteurs jouant un rôle dans l'eutrophisation.
8. Lac Léman : concentration moyenne pondérée et stock de phosphore total.

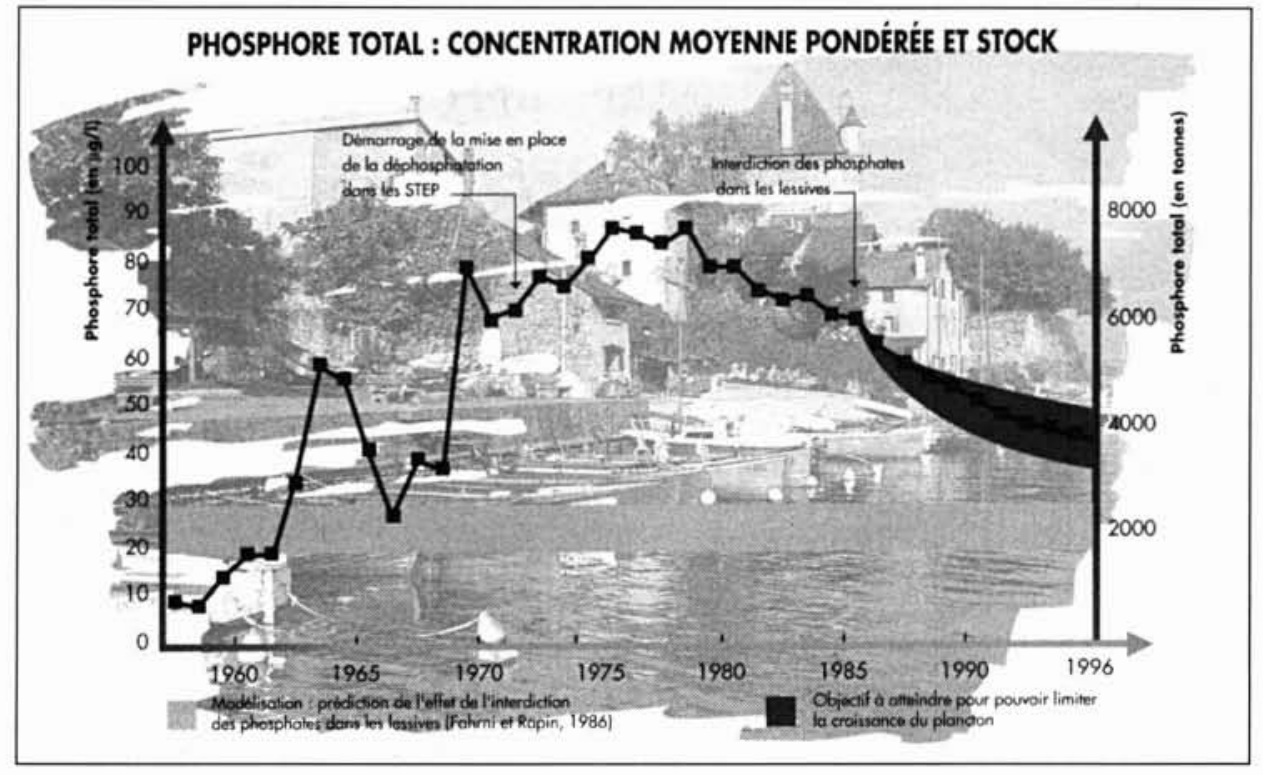


On détaille ci-après par grand axe quelques points forts de ces stratégies complémentaires.

\section{- 4.2 Fondements d'une stratégie de maîtrise}

\subsection{1 abaisser le niveau des importations}

- S'agissant des lessives, il ne saurait être question d'entrer dans le débat sans fin des "vertus écologiques " comparées des lessives avec et sans phosphates.

On se bornera à rappeler, en s'appuyant sur l'exemple du lac Léman vis-à-vis duquel les autorités fédérales suisses ont adopté et appliqué à compter du ler juillet 1986 une ordonnance interdisant les TPP dans les produits de lavage du linge et des textiles, le bénéfice incontestable qui en a résulté au plan de la qualité de l'eau, comme en atteste la figure 8 [8].

On a constaté :

- une baisse de moitié en 10 ans du phosphore dans le lac,

- une baisse corrélative de la biomasse algale,

- le retour d'espèces d'algues de milieu non ou peu perturbé,

- une baisse de la consommation en oxgène du fond et du relargage du phosphore par les sédiments,

- une recolonisation des sédiments par des vers de milieux propres,

- des concentrations toujours très faibles et sans augmentation de NTA et EDTA.

C'est incontestablement une mesure qui aurait les meilleurs effets dans notre pays. On rappellera que $20000 \mathrm{t}$ de phosphore des lessives équivalent à 2,3 $\mathrm{Mt}$ de matière végétale potentielle.

- En matière d'alimentation animale, l'optimisation de la ration des monogastriques (porcs et volailles) et des bovins est une voie de progrès incontestable pour la réduction du phosphore des déjections. C'est un point fort du CORPEN [7].

- pour les monogastriques, trois axes de progrès sont actuellement développés :

a) l'adaptation des apports aux besoins, par fractionnement de l'alimentation selon l'âge et le stade physiologique (alimentation biphase des porcs à l'engraissement et des truies gestantes, qui permet une réduction de 15 à $20 \%$ par rapport aux pratiques alimentaires standard ; alimentation multiphase, avec une réduction supplémentaire de 10 à $20 \%$ ),

b) amélioration de la digestibilité du phosphore de la ration, par recours à des matières premières à forte activité phytasique ou à des phytases d'origine microbienne,

c) amélioration des performances des animaux, par la génétique, l'alimentation et la conduite de l'élevage,

- pour les bovins, une optimisation de la ration est nécessaire mais ne suffit pas. Il faut raisonner à l'échelle du troupeau, de l'exploitation et des surfaces mises en culture. On rejoint la notion de recyclage développée ci-après.

- Pour ce qui est des engrais de synthèse, il s'agit de se rapprocher d'un optimum fourniture/besoins générateur de pertes réduites, sachant qu'en de multiples situations, les sols sont déjà saturés en phosphore mobilisable, ce qui permet d'envisager de progresser par une connaissance meilleure des besoins des plantes et de l'ensemble des fournitures du sol, réduisant la nécessité d'apports complémentaires, ainsi qu'on l'a constaté dans le chapitre 3.2.4.

Le raisonnement de la fertilisation des cultures est, toutes sources de phosphore confondues, un axe stratégique fort à conduire au niveau de l'exploitation agricole.

\subsubsection{Soustraire les milieux sensibles aux effets des rejets directs en phosphore}

C'est la base de la stratégie qui prévaut en matière d'eaux résiduaires urbaines, en déclinaison de la directive de 1991 qui a défini le principe de la délimitation de zones sensibles à l'eutrophisation. Elle y préconise :

- un rendement minimum d'élimination du phosphore total de $80 \%$ ou

- une concentration moyenne annuelle des rejets domestiques de $/ 2 \mathrm{mg} / \mathrm{l}$ (rejet de 10000 à 100000 eh)

$11 \mathrm{mg} / \mathrm{l}$ (plus de $100000 \mathrm{eh}$ )

C'est le domaine privilégié d'une stratégie de maîtrise fondée sur le rapport N/P et la théorie du facteur limitant qui s'appuie sur l'un des plus importants concepts régissant les rapports entre le phosphore et la plante et qui fut énoncé par Liebig en 1855 sous le nom de loi du minimum. Il stipule que la croissance d'une plante peut être limitée par le nutriment le plus déficient par rapport à ses besoins [9].

Selon ce principe, les termes de la stratégie s'expriment comme suit

si N/P eau > N/P tissus, le phosphore disparaît en premier, il est limitant

si N/P eau < N/P tissus, l'azote disparaît en premier, il est limitant.

On a vu au paragraphe 1 , les valeurs moyennes du rapport $\mathrm{N} / \mathrm{P}$ des tissus végétaux.

Mais cette notion a un fondement strictement physiologique. Elle ne s'apparente pas forcément à la notion de facteur de maîtrise à fondement opérationnel et qui se définit comme le facteur sur lequel une action est techniquement et économiquement possible pour le rendre limitant.

Les considérations parfois rencontrées de “ zones limitées par l'azote ou par le phosphore " [10] n'ont donc pas de réelle signification ni fondamentale, ni pratique. On peut seulement dire que les milieux influencés se différencient par des espèces d'algues et/ou de macrophytes qui présentent des exigences physiologiques différentes par rapport à l'azote et au phosphore.

Ainsi, reprenant l'exemple des proliférations de macroalgues vertes du littoral breton (ch.2.3) il est souvent dit que l'azote des lisiers de porc est à l'origine de ces manifestations.

On remarque d'abord que par référence au concept de facteur limitant ci-dessus, le lisier de porc a la composition suivante [11] :

$$
\begin{aligned}
& \mathrm{NK}=5 \mathrm{~g} / \mathrm{l} \\
& \begin{array}{l}
\text { dont } \mathrm{N} \mathrm{NH}_{4}=4 \mathrm{~g} / \mathrm{l} \\
\text { La valeur du ratio N/P en poids est donc de } \underline{3.8} .
\end{array}
\end{aligned}
$$

Les ulves, responsables des marées vertes constatées, présentent un rapport N/P en atomes de 7, soit 3,2 en poids. On se trouve donc dans la situation N/P lisier $>$ N/P tissu, ce qui milite en faveur du phosphore comme élément légèrement limitant.

Par ailleurs, la maîtrise de l'azote est difficile, coûteuse et aléatoire quant aux risques de retour au milieu : elle s'appuie en effet sur sa volatilisation, après nitrification-dénitrification.

Ainsi même dans ces situations extrêmes d'effluents et d'algues à faible valeur relative du rapport N/P, il apparaît 
que la maîtrise du phosphore présente les meilleures garanties d'efficacité.

[5] :

C'est pourquoi, il y a lieu de réaffirmer le principe suivant

- le phosphore : facteur limitant en eau douce et facteur de maîtrise dans tous les milieux,

- I'azote : parfois facteur limitant mais rarement facteur de maîtrise.

Ceci posé, il y a lieu d'examiner les conséquences, sur le phénomène de l'eutrophisation, de la disposition arrêtée en zone d'exédent structurel qui consiste, au-delà d'une certaine quantité d'effluent produite, à en abaisser la teneur en azote par un traitement approprié. Vue sous l'angle de la seule problématique des nitrates, elle risque d'accroître considérablement la teneur en phosphore de l'effluent traité, avec des conséquences mal cernées sur son incidence possible sur les milieux aquatiques.

On notera pour finir que cet axe n'a pour effet qu'un transfert de support du phosphore, du rejet liquide aux boues résiduaires, que l'on va demander aux agriculteurs de gérer, dans un contexte le plus souvent excédentaire considéré à l'échelle de leur exploitation.

\subsubsection{Améliorer les performances de l'exploitation agricole}

C'est la voie privilégiée du recyclage du phosphore. Mais le pilotage en est difficile, ainsi qu'il ressort du schéma simplifié figure 9 (Gueguen, [7]), illustrant les termes d'un bilan de phosphore selon le type d'animal et de régime alimentaire. L'objectif visé est d'ajuster au mieux les apports, par importation et par les fourrages, aux " sorties ", elles-mêmes réutilisées (déjections) de manière optimale.

Hors les situations fatalement excédentaires, telles que les élevages de monogastriques en hors sol, il apparaît qu'un raisonnement affiné de la production de fourrages et de la valorisation des déjections produites sur l'exploitation pourrait le plus souvent dispenser de toute fertilisation par voie externe à l'exploitation (engrais industriels notamment).

Mais cela suppose l'évaluation précise des surfaces nécessaires à la production des fourrages consommés par les animaux, en même temps que des besoins stricts des cultures au regard du phosphore. Pour eux, des références existent, qui sont de plus en plus précises (normes COMIFER) et il suffirait de les appliquer. L'optimisation se rapporte donc au triplet " surfaces fourragères - besoins des cultures - effectif du cheptel " dont les éléments interagissent.

Cependant, cette optimisation est toute théorique et est rendue plus complexe par la nécessité d'intégrer, dans les plans de fumure à l'exploitation, des constituants externes (boues d'épuration) ou encore des effluents animaux qui, s'ils ont été traités, sont proportionnellement enrichis en phosphore.

En de nombreuses situations toutefois, le raisonnement succinctement évoqué ci-dessus, qui se traduit par l'élaboration de plans de fumure pluriannuels détaillés, peut être une voie prometteuse de réduction des pertes de phosphore à l'échelle de l'exploitation.

\subsubsection{Confiner les flux de phosphore}

Cet axe stratégique vise à empêcher le phosphore provenant de sources diffuses comme ponctuelles de rejoindre les milieux aquatiques où il exerce ses effets néfastes.
S'agissant des sources diffuses, dont la caractéristique est d'impliquer de manière majeure l'intermédiaire " sol ", une gestion appropriée du territoire peut permettre d'en réduire les effets. Dans les situations qui cumulent les facteurs de risque (secteur à pente marquée ; forte teneur en phosphore des sols), un aménagement du territoire consistant à " complexifier le trajet de l'eau " et à créer des zones-tampons, des milieux humides intermédiaires entre la source et le milieu récepteur et où l'excès de production végétale peut même être à l'origine d'un gain patrimonial valorisant, est une voie à recommander pour limiter les conséquences de l'eutrophisation.

En matière de rejets ponctuels, de station d'épuration par exemple, la mise en place de drains " naturels " en terre battue par exemple, et non rectilignes, peut favoriser une réutilisation partielle par la végétation s'y développant, du phosphore émis avant qu'il parvienne avec écoulements constitués.

\subsubsection{Traiter au cas par cas les sédiments de rivières et de lacs}

Cet axe est lié directement à ce qui a été dit sur l'inexorable augmentation du stock de phosphore dans l'environnement depuis l'avènement de l'ère industrielle, l'intensification de l'activité agricole et l'accroissement de l'hygiène.

Cela a deux conséquences :

1 - la maîtrise des macrophytes et des algues fixées par la seule réduction des flux émis quelle qu'en soit l'origine, est illusoire. Les stocks en place de phosphore dans les sédiments sont suffisamment importants pour entretenir l'eutrophisation par les végétaux enracinés sur une longue période.

2 - cette accumulation peut agir comme une bombe à retardement, dans le fond des lacs en particulier, car le phosphore en place peut être résolubilisé dans l'eau surnageante, lorsque les conditions du milieu deviennent réductrices. C'est le phénomène de relargage susceptible d'ensemencer les couches productives de surface par la circulation interne au plan d'eau.

Cette caractéristique d'ordre historique ne doit pas décourager pour autant dans l'objectif stratégique de réduction des flux émis dont les effets se répercuteront à terme sur les stocks. Il faut en avoir conscience cependant et intervenir au cas par cas pour limiter l'effet potentiel qui lui est imputable.

A ce niveau, les considérations de coût jouent un rôle important. Néanmoins une série de techniques est désormais à disposition pour inactiver le phosphore des sédiments des rivières et des lacs, qui peuvent être mises en œuvre si le danger qu'il représente est reconnu.

Elles font appel à des procédés physiques et physicochimiques dont le choix dépend du contexte [3].

On notera pour finir que la manifestation de l'eutrophisation est une source sans fin de réensemencement, par le phosphore, du milieu où elle se produit, et non pas une voie de son élimination, du fait de l'alternance des cycles naissance/mort et prélèvement/restitution des substances nécessaires à la production primaire.

\subsubsection{Aménager et entretenir les cours d'eau}

On a vu au paragraphe 4.2.4 l'effet positif lié à la reconstitution de milieux annexes pièges à phosphore. Cet axe stratégique peut être complété par une intervention judicieuse sur les cours d'eau, visant à intervenir sur des facteurs de régulation de l'eutrophisation autres que les nutriments. 


\section{Bilan de phosphore selon le type d'animal et le régime alimentaire}

Alors que la proportion moyenne de phosphore, émis par l'animal sous forme de déjections, susceptible de retourner au sol varie peu et est souvent proche en pratique des $3 / 4$ du phosphore ingéré total, la proportion de phosphore ingéré provenant du sol de l'exploitation (fourrages) est très variable, allant de 0 à 1 . Les quatre exemples du schéma 1 concernent dans l'ordre :

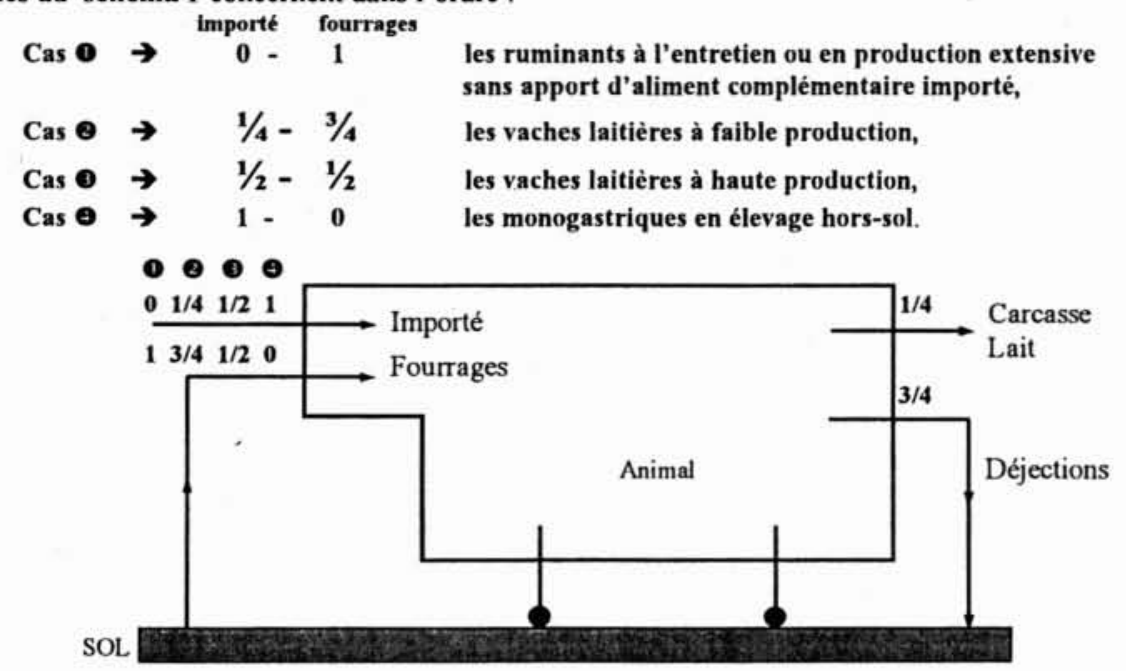

Schéma 1: bilan de phosphore (au niveau de l'exploitation) selon le type d'animal et le régime alimentaire (Guéguen, 1997)

Alors que dans les cas $\mathbf{0}$ et $\boldsymbol{\Theta}$, le phosphore restitué au sol par les déjections ne suffit pas (0) ou tout juste (ब) à compenser le phosphore prélevé par les fourrages, dans les cas Bet $\boldsymbol{\theta}$, il compense le phosphore prélevé par les fourrages et pourrait dispenser de toute fertilisation phosphatée minérale.

Le cas 3 est illustré par le schéma 2 , à travers l'exemple d'une vache laitière produisant $7000 \mathrm{~kg}$ de lait par an et ingérant $22 \mathrm{~kg}$ de $\mathrm{P}$ par an dont $13 \mathrm{~kg}$ en provenance des fourrages de l'exploitation. Environ $7 \mathrm{~kg}$ de P sont exportés ( 6 dans le lait et 1 dans la masse corporelle réformée au bout de 5 ans), tandis que $15 \mathrm{~kg}$ sont restitués par le fumier. Le bilan de phosphore au niveau des sols de l'exploitation (15-13) est donc positif de 2 kg de $P$ par an et par vache.

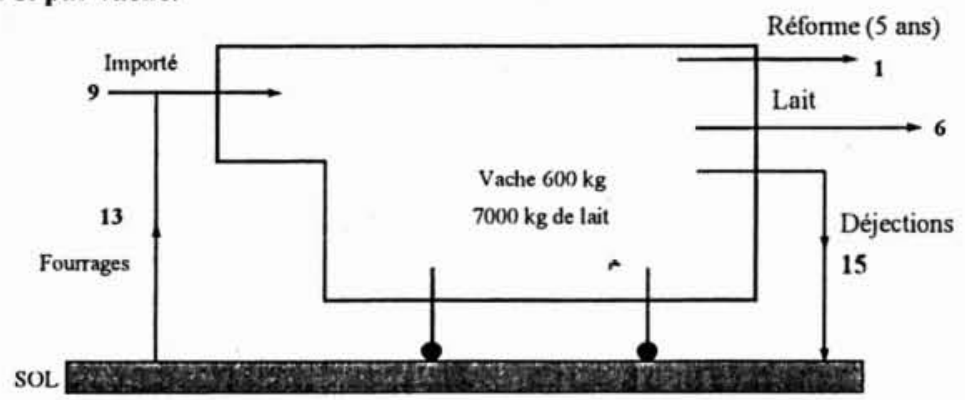

Schéma 2 : bilan de phosphore (au niveau de l'exploitation) d'une vache laitière en $\mathrm{kg}$ de $\mathrm{P}$ par an (Guéguen, 1997).

A titre de comparaison, pour illustrer le cas 0 du schéma 1, pour un "porc charcutier produit " ne consommant que des aliments importés, le bilan de phosphore au niveau des sols de l'exploitation, est de $\mathbf{3} \mathbf{~ k g}$ de $P$ par place de pore charcutier par an ${ }^{18}$

9. Bilan de phosphore selon le type d'animal et le régime alimentaire. 
Toute forme d'intervention sur les cours d'eau sensibles à l'eutrophisation doit prendre en compte ce risque de manière volontaire.

A cet effet, la connaissance de la biologie et des exigences écologiques des espèces végétales en cause, notamment des espèces fixées, apparaît comme tout à fait déterminante pour l'adaptation de l'intervention dans le sens de la maîtrise des conditions physiques nécessaires à leur croissance [12].

Elle pourra concerner tout autant les conditions de l'éclairement (maintien sélectif d'un ombrage approprié) que l'optimisation de la vitesse du courant ou de la ligne d'eau (n'autorisant pas la fixation racinaire) ou l'aménagement des berges pour en accroître le rôle-tampon.

Il s'agit là d'une donnée essentielle de la politique de restauration, d'entretien et de mise en valeur des milieux aquatiques à développer en fonction de l'évolution des connaissances sur les végétaux aquatiques.

\section{RÉFÉRENCES BIBLIOGRAPHIQUES}

[1] " Origine domestique des apports en phosphore ". P. Balland - 34 pages, colloque AIDEC " Le phosphore, ses dérivés et leur comportement dans le milieu naturel " - Dijon - novembre 1988

[2] " Phosphate" - Brochure du Centre Européen d'Etudes des Polyphosphates (CEEP) - 16 pages - 1997
[3] " Manuel de limnologie appliquée " - G. Barroin INRA - Texte non publié - 168 pages + annexes.

[4] " Le phosphore en milieu marin ". A Aminot - 23 pages - Colloque AIDEC sus-cité.

[5] " Eutrophisation des milieux aquatiques : bilan des connaissances et stratégies de lutte ". Note technique SDAGE $\mathrm{n}^{\circ} 2$ - Comité de bassin et Préfet Coordonnateur de bassin Rhône-Méditerranée-Corse - décembre 1996 - 29 pages.

[6] "Inventaire des ulves en Bretagne - Année 1997 - Rapport de synthèse " - IFREMER et Agence de l'eau Loire-Bretagne - 18 pages + annexes.

[7] Programme d'action concemant la maîtrise des rejets de phosphore provenant des activités agricoles - CORPEN 1998.

[8] " Lessives, phosphates et eutrophisation des eaux " - Etudes Inter Agences $\mathrm{n}^{\circ} 39-1997-26$ pages.

[9] " La pollution des eaux stagnantes par les phosphates - Controverses (pseudo) scientifiques et (absence de) décisions politiques " - G. Barroin Environnement, Science et Politique non daté - 89-115.

[10] " Eutrophisation des eaux côtières " - J.L. Mauvais, A. Menesguen, M. Merceron., P. Lassus, Ifremer - Bulletin de I'Agence de I'Eau AdourGaronne n ${ }^{\circ} 45-1990 / 12-18$.

[11] " La gestion des lisiers de porcs associant le traitement en zone d'excédent structurel " - J. Coillard - Cemagref 1997 - 23 pages + annexes.

$[12]$ “ Biologie et écologie des espèces végétales aquatiques proliférantes en France - Synthèse bibliographique " - Etudes Inter Agences - septembre 1997 - 280 pages + annexes. 